\title{
Techniques to Prevent Bedsore in Physically Disabled Patients
}

\author{
K. Janani, M. Poornima Devi, S. Mercy Golda, M. Anisha, G. Vishnuvarthanan, S. Sakthivel
}

\begin{abstract}
Inspite of improvements in medical industry and healthcare, pressure ulcers prevention remains high in hospitalized patients. These pressure ulcers prevails mostly in the patients who stays in hospital for long-term, assisted living at home, paralysed patients and with other physical disorders excluding of their diagnosis and health care needs. Pressure ulcers are known as a kind of skin lesion which influences the patient's integrity of life and their caregiver become an significant reason of morbidity and in some aspects increase in the mortality. Managing and treating pressure ulcers are too costly. Though many devices have been designed with the aim of pressure ulcer prevention and cure but most of the existing technically complex devices have been exposed to be no more efficient and cost effective. Prevalence of these ulcers can be achieved by reducing pressures at bony prominences since pressure ulcers are high-cost and high adverse event. The objective of this manuscript is to review recent articles, with particular emphasis on prevention of bedsore in physically disabled patients.
\end{abstract}

Keywords : Prevalence, Pressure Ulcers, Paralyzed Patients, Mortality, Bony Prominences, Pressure Elevation.

\section{INTRODUCTION}

Pressure Ulcers (PU) are affected by large number of individuals. Pressure ulcers are described as a localized damage to dermal area or to the underneath tissue which is frequently happens over bony prominences. There are various stages of pressure ulcers which are formed due to the heavy pressure on layers of skin or a blend of pressure and cut off to a specific location. The phases are classified in to non-blanchable erythema, incomplete or full skin loss and unstageable injury. This disease has a high mortality rate and it is costlier one. Pressure ulcers are preventable, but the patients and the care providers should provide a good amount of effort to diminish the occurrence. There are many factors which boost the occurrence of PU in all people. They are (a) wheelchair utilization, (c) urinary incontinence, (d) amputation over the knee, (e) Current operation, (g) obesity, (h) inability to position change,(i) residence in a nursing home. PU is a disease which is avoidable, but severe problems may take place when it is not prevented or treated.

Revised Manuscript Received on December 16, 2019.

* Correspondence Author

K. Janani, Biomedical Engineering, Kalasalingam Academy of Research and Education, Krishnankoil, India.

S. Mercy Golda, Biomedical Engineering, Kalasalingam Academy of Research and Education, Krishnankoil, India.

M. Poornima Devi, Biomedical Engineering, Kalasalingam Academy of Research and Education, Krishnankoil, India.

Anisha M*, Biomedical Engineering, Kalasalingam Academy of Research and Education, Krishnankoil, India. Email: anisha@klu.ac.in

G. Vishnuvarthanan, Biomedical Engineering, Kalasalingam Academy of Research and Education, Krishnankoil, India.

S.Sakthivel, Biomedical Engineering, Kalasalingam Academy of Research and Education, Krishnankoil, India.
Persons who stay in same position for a prolonged duration will develop ulcers. Reducing the incidence and persistence of PU will lead to positive social amend by eliminating the morbidity and the economic crisis on affected patients and the health care system from the Centers for Disease. Control and Prevention stated an important information on PU that is Long-term care residents \& patients of 64 years of age and older have $14 \%$ of developing pressure ulcer and less than one year stay has $16 \%$ of developing pressure ulcer risk This project was intended to decrease and prevent PU with the help of pressure elevation for physically disabled.

\section{RELATED WORKS}

Prevention of bedsores or pressure ulcer consists of various techniques for preventing it and also eradicating it with the help of treatments and many devices were also designed. Some of the prominent related works are discussed as follows.

In [1] Syed aziz shah and nan zhao (2016) outlined the concept of automated body posture estimation which used fine-grained channel state information and it is crucial to diminish the occurrence of PUs or bedsores on skin which specified that utilizing the leaky coaxial cable with low-cost wireless devices. Roma Rattan and Sadasivan Jagdish , (2008) [2] aimed to underlined the importance of bedsores by reviewing the current and past knowledge with help of practical implicationsIn [3] Tatiana V. Boyko and Michael T. Longaker , 2018 discusses that the Pressure ulcers represent a large and growing biomedical burden to society. Understanding prevention and treatment is necessary for wound care specialists. Novel dressings and therapies have not shown increased benefit over others, but they have utility based on specific patients

In [4] Wearable wireless devices by Devdip Sen and John McNeill, (2018) described a powered sensor system is used to alert the caregiver the potentially contact pressure surfaces at different locations with the help of machine learning. In [5] Rasoul Yousefi and Miad Faezipour, (2011) discussed on the aetiology, risk management, interventions and treatment measures of pressure ulcers.In [6] Deborah Behan and Alan Bowling, 2016 presents the preliminary design of the force bed for performing manipulation, and the configuration of the patient and the bed were determined with help of a feedback from group of sensors.

In [7], a subject dependent algorithm were proposed by F. Knoefel and K. Rockwood (2016), and which is capable ofdetecting automatically when the pressure points have been relieved without any user inputs . 


\section{Techniques to prevent Bedsore in Physically Disabled Patients}

In [8] the Rasoul Yousefi and Miad Faezipour,(2015) developed a software-hardware platform that addressed the pressure ulcers and this is their main focus. The information from the various sensors is collected and the data were analyzed. The actuators in the bed provide periodical adjustment to the whole body by redistributingthepressure In this paper [9] Jill A. David (2014) discussed the bed sore management and surgical methods treating bed sores. In [10] the adverse effect of the pressure ulcers, measuring the pressure ulcer accuracy and staffs management was addressed by Dan Berlowitz, (2011) to reduce its prevalence. In [11] Ashkan Javaheri and Daniel Bluestein, (2015) discussed the anatomy of skin and pressure ulcer were studied and assessment, prevention and management of the bed sore through staffing, repositioning of the patients, support surface adjustment and treatment for pressure ulcer were discussed.

In [12] Force Sensing Resistors were addressed by S. Pereira and R. Simoes, (2014). According to the sensing system feedback and pressure relief need pressure ulcers were reduced. In [13] a prevention system for monitoring pressure ulcer risk factor were designed by Dieter Hayn et al, 2015 with the help of accelerometer and pressure sensors.Sensors are employed for identification of repositioning of the patient and the corresponding information are then transmitted to the tablet for analyzing.

In 2017 S. Raja et al, [14] presented a method which is proposed to reduce the risk of Hospital Acquired Pressure Ulcers. The proposed method consists of checker board mattresses which automatically change its position according to the mobility and activity of the patient by using the Braden scale assessment tool and it requires the appropriate sensors to monitor the mobility and activity of the patient. The patient's position is regularly monitored and automatically position changes is done and updated to the doctor's and the hospital's system, with the help of centralized system for monitoring

In [8] \& [15] R. Yousefi et al, (2011) developed a software and hardware system for pressure ulcer. The system gains the information from sensors which is placed inside the bed and analyzes the data and commands the actuators to change its surface periodically for redistributing the pressure hence a support system is created and it increases the ability of a caregiver.In [16] Raiphale et al , (2018) outlined an antibedsore bed that prevents the occurrence of pressure ulcers by alleviating contact pressure exerted on a patient due to contact in a bed. In this [16], a set of air bags are installed below a hospital bed to alleviate the bed and it is used to alleviate a patient's head, hip, thighs and heels which are generally the bedsores occurring areas. The air is filled in the air bags using air compressor by using controller unit. This reduced the effort of the person to tilt the patient manually. In [6] \& [17] Shahnavaz Eilbeigi Haiying et al 2017 presented a Force bed concept which can manipulate a object . The automated system were designed to do the required task to prevent pressure ulcers The hardware is divided in to two main systems, which is used to perform manipulation, and to provide feedback for determining the patient and the bed configuration. A controller was used to manipulate the patient with the help of sensors

In [18] a flexible pressure monitoring system was constructed by Marcus Yip et al, (2009) for PU prevention.
The resolution of each sensor ranges more than $2-\mathrm{mmHg}$ and it ranges up to $50-\mathrm{mmHg}$. The real time pressure data was monitored with a help of GUI software and Matlab was used for further processing.

Table. 1. Summary of the Existing Methods

\begin{tabular}{ll}
\hline \multicolumn{1}{c}{ Work } & \multicolumn{1}{c}{ Technique Used } \\
\hline & $\begin{array}{l}\text { Body posture detection utilizing the } \\
\text { leaky coaxial cable with low-cost } \\
\text { wireless devices. }\end{array}$ \\
$\begin{array}{l}\text { Syed aziz shah, Nan } \\
\text { zhao (2016) }\end{array}$ & $\begin{array}{l}\text { Wearable wireless device - a powered } \\
\text { sensor system is used with the help of } \\
\text { machine learning to alert caregiver }\end{array}$ \\
$\begin{array}{l}\text { Devdip Sen, John McNeill, } \\
\text { (2018) }\end{array}$ & $\begin{array}{l}\text { Subject dependent algorithm } \\
\text { proficient of automaticallyidentifying } \\
\text { PU locations. }\end{array}$ \\
\hline $\begin{array}{l}\text { F. Knoefel , } \\
\text { K. Rockwood } \\
\text { (2016) }\end{array}$ &
\end{tabular}

Rasoul Yousefi,Miad

Faezipour, (2015)

\section{Jill A. David}

(2014)

Dan Berlowitz, (2011)

Dan Berlowitz, (2011)

S. Pereira, R. Creation of actuation system based on

Simoes , (2014)

Dieter Hayn et al,
( 2015)

S. Raja et al, (2017)

Raiphale, et al, (2018)

Raiphale, et al, (2018)

Cristina Díaz et al , ( 2017)

Developed software-hardware platform that addresses the pressure ulcers with the help of bed actuators

Surgical methods treating bed sores.

Addresed adverse effect of the pressure ulcers, measuring the pressure ulcer accuracy and staffs management force sensing resistor (FSR)

Identification of relocating of the patient lying on the mattress.

Checker board mattresses which automatically change its position according to the mobility

Anti-bedsore bed that prevents the occurrence of pressure ulcers by alleviating contact pressure exerted on a patient with the help of airbags

Electronic system with an sufficient sensor well proficient of scrutinizing the state of the pressure ulcer.

\begin{tabular}{|c|c|c|}
\hline $\begin{array}{l}\text { Marcus Yip et al, } \\
(2009)\end{array}$ & $\begin{array}{l}\text { Developed } \\
\text { system for F }\end{array}$ & $\begin{array}{l}\text { a flexible } \\
\text { pressure monitoring } \\
\text { oidance. }\end{array}$ \\
\hline
\end{tabular}

In [19] preliminary concepts of prevention of pressure ulcers were discussed by Cristina Díaz et al , (2017) with the help of a sensor-based system and in the moto to reduce the occurrence in hospitalized patients with the help of improving the detection in pressure ulcer risk areas. The electronic system was designed with an adequate sensor in order to analyze the state of the PU. The machine was designed in order that it display the PU along with their symptoms by taking the picture of immobilized area of patients and running it In [20] V. Boyko et al, (2016) delivered the general review about the indications, prevention and treatment of pressure ulcers and also it speaks about the current methodology in practice and its significance. In [21] Barry and Maree et al, (2015) outlined 
the general view about the Pressure ulcers and a negative essence of life, and the older patients are at the risk of forming pressure ulcers. And the difficulties of preventing PUs in geriatrics were addressed clearly. In [22] the complexities of pressure ulcer development and the assessment process were explained by Collier and Mark, (2016) and which is used to spot out the people who are at the danger of developing pressure ulcer. It proves that the use of a risk assessment tool is in forefront to identify an overall pressure ulcer prevention strategy,

In [23] Eunice O. Osuala, 2014 were reviewed the literature and relevant information on the current trend in the prevention and management of pressure ulcers were provided to the nurses to work effectively in pressure ulcer management In [24] Rey-David Palomares and Ojus Rao (2018), designed the Pressure Ulcer Prevention System The system used a gyroscopic sensor and a pressure sensors for data collection and which is mounted on the patient, and it sends warning or reminder to the nurse station if a patient is at risk of developing a pressure ulcer. In [25] Rosa Loikkanen and Mariam Tammi, (2016) outlined the prevention and practices guidelines for PU.

\section{CONCLUSION}

This journal uses double-blind review process, which means that both the reviewer (s) and author (s) identities concealed from the reviewers, and vice versa, throughout the review process. All submitted manuscripts are reviewed by three reviewer one from India and rest two from overseas. There should be proper comments of the reviewers for the purpose of acceptance/ rejection. There should be minimum 01 to 02 week time window for it.

English citation first, followed by the original foreign-language citation [8].

\section{REFERENCES}

1. Shah, Syed Aziz, Nan Zhao, Aifeng Ren, Zhiya Zhang, Xiaodong Yang, Jie Yang, and Wei Zhao. "Posture recognition to prevent bedsores for multiple patients using leaking coaxial cable." IEEE Access 4,pp.8065-8072, 2016.

2. Nayak, Debashish, K. Srinivasan, Sadasivan Jagdish, Roma Rattan, and Vinayaka S. Chatram. "Bedsores:"top to bottom" and "bottom to top"." Indian Journal of Surgery 70, no. 4,pp. 161-168, 2008.

3. Boyko, Tatiana V., Michael T. Longaker, and George P. Yang. "Review of the current management of pressure ulcers." Advances in wound care 7, no. 2,pp 57-67, 2018.

4. Sen, Devdip, John McNeill, Yitzhak Mendelson, Raymond Dunn, and Kelli Hickle. "A New Vision for Preventing Pressure Ulcers: Wearable Wireless Devices Could Help Solve a Common-and Serious-Problem." IEEE pulse 9, no. 6, pp 28-31, 2018.

5. Pressure Ulcers: Prevention, Evaluation, and Management(Rasoul Yousefi,. Miad Faezipour) 2011, Indian Journal of Surgery.

6. Pressure Ulcer Prevention Using Soft, Non-GraspManipulation in a Forcebed(Deborah Behan, Alan Bowling) 2016

7. Bennett, Stephanie L., R. Goubran, Kenneth Rockwood, and Frank Knoefel. "Monitoring the relief of pressure points for pressure ulcer prevention: A subject dependent approach." In 2013 IEEE International Symposium on Medical Measurements and Applications (MeMeA), pp. 135-138. IEEE, 2013

8. A Smart Bed Platform for Monitoring \& Ulcer Prevention (Rasoul Yousefi,.Miad Faezipour) 2015 Indian Journal of Surgery.

9. David, Jill A. "Pressure sore treatment: a literature review." International journal of nursing studies 19, no. 4, pp.183$191,1982$.

10. Berlowitz, Dan, C. VanDeusen Lukas, V. Parker, A. Niederhauser, J. Silver, C. Logan, and E. Ayello. "Preventing pressure ulcers in hospitals: a toolkit for improving quality of care." Agency for Healthcare Research and Quality (2011).
11. Bluestein, Daniel, and Ashkan Javaheri. "Pressure ulcers: prevention, evaluation, and management." American family physician 78, no. 10 (2008).

12. Pereira, S., R. Simoes, J. Fonseca, R. Carvalho, and J. Almeida. "Textile Embedded Sensors Matrix for Pressure Sensing and Monitoring Applications for the Pressure Ulcer Prevention." In 2018 International Conference on Biomedical Engineering and Applications (ICBEA), pp. 1-6. IEEE, 2018.

13. Hayn, Dieter, Markus Falgenhauer, Jürgen Morak, Karin Wipfler, Viktoria Willner, Walter Liebhart, and Günter Schreier. "An eHealth system for pressure ulcer risk assessment based on accelerometer and pressure data." Journal of Sensors 2015.

14. Raja, S., A. Senthil Kumar, N. Priyanka, S. Ramya, and R. Sahana. "A Novel System to Tackle Hospital Acquired Pressure Ulcer Patients."

15. Yousefi, Rasoul, Sarah Ostadabbas, Miad Faezipour, Mehrdad Nourani, Vincent Ng, Lakshman Tamil, Alan Bowling, Deborah Behan, and Matthew Pompeo. "A smart bed platform for monitoring \& ulcer prevention." In 2011 4th International Conference on Biomedical Engineering and Informatics (BMEI), vol. 3, pp. 1362- 1366. IEEE, 2011.

16. A Review on Design and Development of Anti-Bedsore Bed for Patients ( Govind U. Raiphale, Abhishek P. Godse, Kshiteej S. Dhotre, Omkar N.Chakor) 2018.

17. Eilbeigi, Shahnavaz, Haiying Huang, Alan Bowling, and Deborah Behan. "Pressure ulcer prevention using soft, non-grasp manipulation in a forcebed." In 2017 IEEE Workshop on Advanced Robotics and its Social Impacts (ARSO), pp. 1-6. IEEE, 2017

18. Yip, Marcus, David Da He, Eric Winokur, Amanda Gaudreau Balderrama, Robert Sheridan, and Hongshen Ma. "A flexible pressure monitoring system for pressure ulcer prevention." In 2009 Annual International Conference of the IEEE Engineering in Medicine and Biology Society, pp. 1212-1215. IEEE, 2009.

19. Díaz, Cristina, Begoña Garcia -Zapirain, Cristián Castillo, Daniel Sierra-Sosa, Adel Elmaghraby, and Paul J. Kim. "Simulation and development of a system for the analysis of pressure ulcers." In 2017 IEEE International Symposium on Signal Processing and Information Technology (ISSPIT), pp. 453-458. IEEE, 2017.

20. Review of the Current Management of Pressure Ulcers (Tatiana V. Boyko, Michael T. Longaker and George P. Yang ) 2016 , wound healing society (WHS)

21. Barry, Maree, and Linda Nugent. "Pressure ulcer prevention in frail older people." Nursing Standard (2014+) 30, no. 16 (2015): 50

22. COLLIER, MARK. "Pressure Ulcer prevention: Fundamentals for best practice." acta medica croatica 70, no. Suplement 1 (2016): 3-9.

23. Osuala, Eunice O. "Innovation in prevention and treatment of pressure ulcer: Nursing implication." Tropical Journal of Medical Research 17, no. 2 (2014): 61.

24. Palomares, Rey-David, and Ojus Rao. "Pressure Ulcer Prevention System." (2018)

25. Loikkanen, Rosa, and Mariam Tammi. "Pressure ulcer prevention and its implementation in practise: a literature review." (2016)

\section{AUTHORS PROFILE}

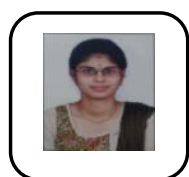

Ms. M. Poornima Devi has received her B.Tech Biomedical Enineering from Kalasalingam Academy of Research in 2019.

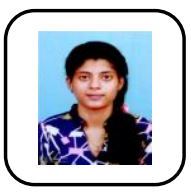

Ms.S. Mercy Golda has received her B.Tech Biomedical Enineering from Kalasalingam Academy of Research in 2019.

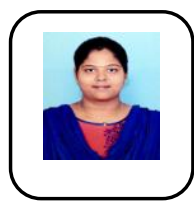

Ms. K. Janani has received her B.Tech Biomedica Enineering from Kalasalingam Academy of Research in 2019. 
M. Anisha received her BTech in BioInformatics from Sathyabama University, Chennai in 2009, MTech in BioInformatics from Sathyabama University in 2011, and $\mathrm{PhD}$ in the area of Biosignal Processing from Noorul Islam Centre for Higher Education in 2018. At present, she is working as an Assistant Professor in the Department Biomedical Engineering, Kalasaligam Academy of Research and Education, Krishnankoil, India.

Dr. G. Vishnuvarthanan, born in 1986, has research stints in the avenues of medical image processing and artificial intelligence. He was awarded $\mathrm{PhD}$ in the year 2015 and bachelor's degree in Instrumentation and Control Engineering by 2007, and Master's Degree in VLSI by 2009. He has more than ten years of teaching and research experience and has his affiliation as Associate Professor with the Department of Biomedical Engineering of School of Bio and Chemical Sciences in the Kalasalingam Academy of Research and Education, Tamilnadu, India

Sakthivel Sankaran received B-Eng. in Electronics and Communication Engineering, May 2013 and M.Tech in (Biomedical Engineering) at VIT University, Vellore, May 2015. Currently working as an Assistant Professor in Biomedical Engineering department at Kalasalingam Academy of Research and Education, Krishnankoil. His research interests are Biomechanics, Rehabilitation Engineering and Biomedical Instrumentation. He has so far published more than 09 papers in national and international journals and conferences. He is a Fellow of Indian Society For Technical Education (ISTE), Institute of Electrical and Electronics Engineers (IEEE), Biomedical Engineering Society of India (BMESI) (sakthivelsankaran92@gmail.com). 